\section{(C) OPEN ACCESS}

\title{
Implementation of an endoscopy safety checklist
}

\author{
M Matharoo, ${ }^{1,2}$ S Thomas-Gibson, ${ }^{1,2}$ A Haycock, ${ }^{1,2}$ N Sevdalis ${ }^{2}$
}

\section{- Additional material is published online only. To view please visit the journal online (http://dx.doi.org/10.1136/ flgastro-2013-100393) \\ ${ }^{1}$ The Wolfson Unit for Endoscopy, St. Mark's Hospital, Harrow, UK \\ ${ }^{2}$ Department of Surgery and Cancer, Imperial College, London, UK}

\section{Correspondence to}

Dr Manmeet Matharoo, The Wolfson Unit for Endoscopy, St. Mark's Hospital, Harrow, Middlesex HA1 3UJ, UK; m.matharoo@imperial.ac.uk

Received 23 September 2013 Revised 2 December 2013 Accepted 3 December 2013 Published Online First

19 December 2013

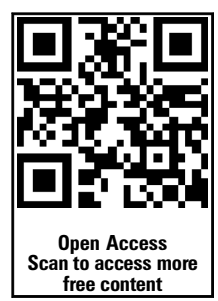

CrossMark

To cite: Matharoo M,
Thomas-Gibson S, Haycock A,
et al. Frontline
Gastroenterology
2014;5:260-265.

Thomas-Gibson S, Haycock A, Gastroenterolog 2014;5:260-265

\begin{abstract}
Patient safety and quality improvement are increasingly prioritised across all areas of healthcare. Errors in endoscopy are common but often inconsequential and therefore go uncorrected. A series of minor errors, however, may culminate in a significant adverse event. This is unsurprising given the rising volume and complexity of cases coupled with shift working patterns. There is a growing body of evidence to suggest that surgical safety checklists can prevent errors and thus positively impact on patient morbidity and mortality. Consequently, surgical checklists are mandatory for all procedures. Many UK hospitals are mandating the use of similar checklists for endoscopy. There is no guidance on how best to implement endoscopy checklists nor any measure of their usefulness in endoscopy. This article outlines lessons learnt from innovating service delivery in our unit.
\end{abstract}

\section{BACKGROUND}

More than a decade ago, the landmark Institute of Medicine's report, 'To Err is Human: Building a Safer Healthcare System, ${ }^{1}$ revealed that between 44000 and 98000 patients die each year in the USA as a result of preventable medical error. The cost of this is estimated at \$17-29 billion per year. This was mirrored in the UK: a retrospective review of patients records by Vincent et al ${ }^{2}$ showed approximately $10 \%$ of UK patients underwent a medical error, with half deemed to be preventable and a third of patients suffering significant disability or death. These errors within the National Health Service (NHS) amount to a cost of $£ 1$ billion a year in extra bed-days alone. ${ }^{2}$ These findings are corroborated internationally by a recent systematic review. ${ }^{3}$

Endoscopy is no exception. In 2004, the Report of the National Confidential Enquiry into Patient Outcome and Death (NCEPOD) 'Scoping our Practice'4 investigated 1818 deaths within 30 days of a therapeutic gastrointestinal (GI) endoscopy. It found that $14 \%$ of endoscopic procedures were inappropriate and 9\% were futile and made 21 recommendations aimed at improving identified deficiencies in organisational structure, training and education, endoscopists' technical skill and patient information and monitoring. Although the recent British Society of Gastroenterology (BSG) audit $^{5}$ shows significant improvements in certain technical aspects such as caecal intubation rates, other aspects of endoscopy patient safety have not been re-evaluated on a large scale.

Several factors make endoscopic procedures increasingly at risk of causing adverse events and patient harm. First, population-based bowel cancer screening exposes healthy, asymptomatic individuals to bowel preparation, sedation as well as an invasive endoscopic procedure, which may also involve high-risk therapy. Second, our ability to perform more numerous and more advanced therapeutic procedures throughout the GI tract has developed the field into much more of a surgical specialty with the associated inherent risks and complications. Surgical specialties have been identified in the evidence base as the most at-risk clinical areas for patient harm ${ }^{3}$ - which is perhaps not surprising given the invasiveness and complexity of the procedures. Other drivers increasing the demand for endoscopic procedures estimated to be $10-15 \%$ per annum ${ }^{6}$ include our ageing population; an increase in conditions requiring endoscopy, such as liver disease and advancing technology allowing endoscopists to perform more and more invasive 'endo-surgical' solutions. ${ }^{7}$

In addition to increased volume of endoscopic procedures, there is also 
increased emphasis on safety at a national level. In 2008, The Department of Health's National Patient Safety Agency issued guidance on a series of so-called 'Never Events' ${ }^{\prime}$ defined as a 'serious but preventable patient safety incidents'. This list started as eight serious incidents resulting in significant harm to patients and expanded to 25 in 2012. Reporting of Never Events is mandatory to encourage learning and implement preventive strategies. There are significant financial disincentives associated with Never Events, as commissioners can recover the cost of the care episode. In 2009-2010, Never Events cost the UK NHS an estimated $£ 3.9$ million. A number of the 25 Never Events are directly relevant to endoscopy:

- Overdose of benzodiazepine during conscious sedation

- Failure to monitor and respond to oxygen saturations during a sedated procedure

- Patient misidentification

- Wrong site surgery (or wrong endoscopic procedure)

- Misplacement of a nasogastric tube

Quality and safety cannot be compromised in our expanding specialty and proactive measures to maintain and improve safety should be sought. Most medical tasks are complex and any intervention involves numerous steps at which an error can occur. In the vast majority of cases, these errors are corrected or compensated for and patients do not come to any harm. However, if errors align and are not captured as the patient goes through the care pathway as in Reason's 's 'Swiss Cheese' phenomenon, significant harm may occur. So, errors can be 'with or without consequence' and near misses may be an opportunity to intervene, thereby avoiding a more serious event in future.

\section{HOW CAN ERRORS BE PREVENTED FROM OCCURRING? A CHECKLIST FOR ENDOSCOPY}

In the past decade, the evidence base on how to prevent errors from occurring in healthcare has expanded dramatically. We now know that errors can be prevented through better team working and safety culture in healthcare, ${ }^{10}$ and that team training and organisational leadership for safety and quality improvement initiatives are important factors. ${ }^{11}$ The safety intervention that has captured the attention of all surgical and interventional specialties is the WHO surgical safety checklist. In 2009, a landmark paper described the development and application across eight hospitals worldwide of the WHO checklist. ${ }^{12}$ This simple tool, a checklist, demonstrated the ability to significantly reduce mortality from $1.5 \%$ before the checklist was introduced to $0.8 \%$ after the checklist implementation.

How can we apply this checklist to endoscopy? The premise of a checklist is to provide an opportunity moments before a procedure starts to check that vital information is shared with the whole team. ${ }^{13}$ As with surgery, a patient will usually have been admitted by a health professional (usually a nurse) outside of the endoscopy room. In some cases, the consent may even have been completed or partially completed by a different member of the team. In almost all cases, there will be endoscopy nurses within the room who have no prior knowledge of the patient. A checklist allows sharing of information pertinent to the individual patient and to their case. It is not meant to be a repeat of the entire admission document.

The key features of an endoscopy checklist to be completed immediately before a procedure starts (ie, the equivalent of the WHO surgical checklist 'Time Out' section) are listed in box 1 . These are neither exhaustive nor mandatory and we take the view that every endoscopy unit ought to develop one that they feel best works for their team. However, effective checklists should be as concise and as user-friendly as possible, ${ }^{14}$ while covering the main issues that could give rise to an adverse event, Never Event or patient safety incident. The proposed checklist is based on observations of errors across endoscopic procedures in our unit and hence is applicable to upper and lower GI endoscopy. The rationale behind each of our suggested parameters is given in box 1

Regarding the practical application of an endoscopy checklist, we believe that it is vitally important that one individual takes the lead for the completion of the checklist and is responsible for ensuring 'Time Out' does occur. This does not necessarily need to be the endoscopist and could equally be the lead nurse. Whoever leads, however, must ensure the entire team are engaged in the process.

As with the WHO surgical checklist, we propose that there is a section to be completed immediately after the procedure and before the patient leaves the room-a 'Sign Out' section. This would include the information listed in box 2.

\section{IMPLEMENTATION OF A CHECKLIST WITHIN THE ENDOSCOPY DEPARTMENT}

Any new intervention requires input from key members of the team to ensure buy-in from all stakeholders. ${ }^{15}$ Each endoscopy unit will have their own ideas about how best to incorporate a checklist into their protocols. Some may choose to have a paperbased version, either as part of the admission booklet or a separate dedicated form. Others may be able to incorporate it into the electronic endoscopy reporting system, some of which have the facility either for all checklist items to be included in their programme or to customise fields to include a checklist.

Once the checklist template has been agreed by the endoscopy users, a period of training and implementation should be planned. Most endoscopy units in the UK have two pools of staff: the endoscopy nurses who are a constant presence in the unit and several (indeed many in some cases) endoscopists from different disciplines (surgical, medical and nursing) who 
Box 1 Key features of a checklist for endoscopic procedures: 'Time Out' (immediately before procedure starts)

\section{Check patient identity}

The patient should be requested to state their name and date of birth. This should be checked against the patient's name band and against any documentation within the room, for example, the notes, patient identity stickers, consent form and the endoscopy reporting system screen. This is always important but particularly so where the list order may have changed; pooled lists are in operation; consent may have been taken by another endoscopist; patients are confused or speak poor English.

2. The core endoscopy team should introduce themselves to the patient

Not only does this reassure the patient but it also breaks down professional boundaries and ensures all members of the team do know each other by name. This facilitates communication particularly in times of difficulty or crisis. Other units may prefer to adopt a whiteboard with team member names listed which is updated at the beginning of each list $+/$ - case.

3. The correct indication and consent is confirmed

The consent form should be checked for signatures of the patient, healthcare professional (countersigned if necessary) and a translator if required. This serves to ensure the whole team are aware of what procedure is to be performed and facilitates the preparation of the case.

4. Confirmation of patient preference for sedation

This should be checked and confirmation that the patient is appropriately monitored prior to administration of sedative drugs (functional intravenous access and oxygen pulse oximetry).

5. Relevant comorbidities are shared

This is not intended to be a repeat of the entire medical history, but important and pertinent history that may be relevant should be verbalised.

\section{Anticoagulant medication}

Confirmation of type and last dose of anticoagulants is critical, particularly for therapeutic procedures.

7. Correctly functioning equipment

Finally, the team should confirm that they have all the necessary equipment required for which every procedure is planned, including kit required in the event of a complication, for example, bleeding.

may come to the unit as little as once per week. A carefully planned implementation programme with suitable notification is a prerequisite for the successful adoption and long-term sustainability of a checklist. ${ }^{15} 16$ A multipronged approach will help and this can be achieved by presenting the evidence for its utility in governance, endoscopy user group meetings,
Box 2 Key features of a checklist for endoscopic procedures: Sign Out (before the patient leaves the endoscopy room)

\section{Check histological samples}

Two people should check that the correct specimen(s) in pot(s) are correctly labelled and the accompanying documentation matches.

9. Check endoscopy report

The report should be an accurate reflection of the procedure (name of endoscopist/supervisor; procedure performed; postprocedure instructions, where relevant).

\section{Clear follow-up plan}

Clear recommendations for surveillance interval, further investigations or treatment duration.

grand rounds and departmental meetings. Simply launching the checklist without training and familiarisation is likely to be unsuccessful as it may become a 'tick-box exercise' if team members do not understand the rationale behind its use.

Ideally we would recommend that several staff are invited to champion the use of the checklist and that they act as leads in the implementation of change. ${ }^{15} 16$ This may include a lead nurse, endoscopist and even trainees. If possible, some or all of these could have dedicated time to support users during the first week(s) of its introduction to help troubleshoot, remind the teams and to facilitate usage. Strong leadership is required from within the nursing and medical team, including from the medical and nursing directors of the institution to establish a change in practice. If done poorly, evidence suggests that the patient may be at more risk, especially if everyone

Box 3 Examples of errors captured and corrected by checklist use in our unit

- Patient in procedure room with no patient identification band

- Incorrect patient details on endoscopy reporting software

- Patient in procedure room with a known drug allergy — picked up during checklist-no allergy alert wrist band

- Incorrect consent form (consent form 4 indicated, form 1 completed)

- Patient required Oesophogo-gastro-duodenoscopy (OGD) and colonoscopy, booked and consented only for colonoscopy-patient alerted team in procedure room and OGD added

- Incorrect patient details on histology sample pot and accompanying paperwork 


\section{Box 4 A checklist for checklist implementation}

Engagement and ownership

- Publicity/'Champions'

- Training plan

- Support for users

- Audit

Feedback

assumes someone else has completed the necessary checks. ${ }^{17}$

Interim feedback (eg, at about 4-6 weeks) after the introduction of the checklist will allow staff to contribute ideas as to how to improve and adapt the checklist. Sharing 'in-house' clinical examples of where the checklist mitigated an error may also facilitate uptake (box 3).

Audit of checklist completion is also advised so that the leads can understand the challenges, target poor uptake and support those who do not appear engaged or who do not see its relevance. Our checklist implementation strategy is summarised in box 4 .

\section{WHAT ARE THE ENABLERS AND BARRIERS TO CHECKLIST IMPLEMENTATION?}

In our unit, a checklist was developed to address common and significant patient safety issues we encountered (figure 1). Sequential multidisciplinary expert focus groups were convened to draft the initial checklist and subsequent versions revised following feedback from team members. The checklist has been in place for over a year now and has since become mandated by the hospital's medical director. We have learnt several lessons in optimising checklist application in the process. In our experience, there are several barriers and enablers to successful implementation of a checklist within an endoscopy department-these are not very different from those that have been identified previously in relation to the WHO surgical checklist. ${ }^{13}{ }^{17} 18$ These are summarised (table 1).

\section{WHAT WILL YOUR PATIENTS THINK?}

One concern may be that patients would be made anxious by being asked repeated questions once in the endoscopy room. In our experience, if it is explained to the patient that this is a final safety check as a

\begin{tabular}{|c|c|c|c|}
\hline \multirow{2}{*}{ 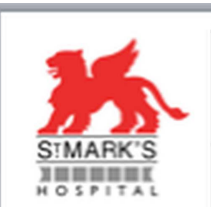 } & \multicolumn{2}{|c|}{ ENDOSCOPY SAFETY CHECKLIST } & \multirow{2}{*}{$\begin{array}{l}\text { प } \\
\text { Affix patient ID label } \\
\square \text { Yes }\end{array}$} \\
\hline & \multirow{11}{*}{ 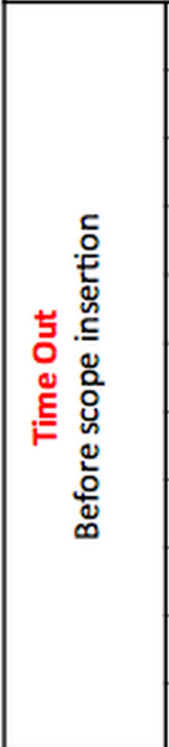 } & Team introduction & \\
\hline & & Patient ID & $\square$ Yes \\
\hline & & Correct screen on reporting software & $\square$ Yes \\
\hline & & Correct procedure & $\square$ Yes \\
\hline & & Indication & $\square$ Yes \\
\hline & & Consent & $\square$ Yes \\
\hline & & Monitoring (IV access / $\mathrm{O}_{2}$ sats) & $\square$ Yes \\
\hline & & Allergies & $\square$ Yes $\square$ No \\
\hline & & Comorbidity & $\square$ Yes $\square$ No \\
\hline & & Anticoagulants & $\square$ Yes $\square$ No \\
\hline & & Correct scope \& kit & $\square$ Yes \\
\hline & \multirow{3}{*}{ 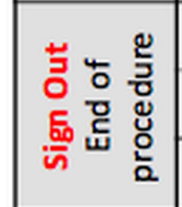 } & Samples \& labelling & $\square$ Yes $\square$ N/A \\
\hline & & Accurate report & $\square$ Yes \\
\hline (d) & & Follow-Up & $\square$ Yes \\
\hline $\begin{array}{l}\text { Wolfson Unit } \\
\text { for Endoscopy }\end{array}$ & \multicolumn{3}{|c|}{ Name (Dr / Nurse) ...............................Date \& Time.....................Signature................................. } \\
\hline
\end{tabular}

Figure 1 Endoscopy safety checklist employed at St. Mark's Hospital. 


\begin{tabular}{ll}
\hline Enablers & Barriers \\
\hline Support from 'external' respected sources (eg, BSG Endoscopy lead) & Lack of evidence from within endoscopy (currently being addressed by \\
Support from other departments familiar with checklist & ongoing work) \\
(eg, anaesthetics, surgery and management) & Professional hierarchy \\
Endorsement from trust medical director & 'Tick-box' exercise \\
Strong leadership from within the endoscopy team; core team & Partial uptake and suboptimal practice tolerated \\
members leading by example & Followership encouraged: following by example (eg, by more junior \\
staff or new team members) & modifications
\end{tabular}

measure to ensure all members of the team in the room know their case and that all documentation matches up, patients are reassured. In auditing, the uptake of the checklist and patients' views should be sought, as part of the patient feedback/satisfaction surveys required for the Global Rating Scale. ${ }^{19} 20$

\section{CONCLUSION}

In a medical world where safety and quality is paramount and under rightful scrutiny, medical error will still occur but will be less well tolerated. A checklist will not prevent every error in endoscopy-it cannot achieve this, nor should it be intended to do so. If it could, however, prevent even a small number of errors and promote an enhanced culture of safety through improved teamwork in the endoscopy room, we strongly believe its use will be justified. In those cases where error does occur and a checklist has not been used, there is likely to be room for criticism. The checklist is a simple, inexpensive, effective tool that has the potential to promote reliable safe endoscopy partly through a system of robust checks, but also by enhancing teamwork in endoscopy.

Contributors Writing, editing and revising manuscript: MM, ST-G, AH and NS. Design, modification and implementation of checklist: MM, AH and ST-G. Training in checklist use: MM, $\mathrm{AH}$ and ST-G.

Funding MM is funded by the NHS Bowel Cancer Screening Programme and the Freemasons Society. MM and NS are affiliated with the Imperial Centre for Patient Safety and Service Quality (http://www.cpssq.org), which is funded by the National Institute for Health Research.

\section{Competing interests None.}

Ethics approval NRES Committee London, Reference 08/ H0719/54.

Provenance and peer review Not commissioned; externally peer reviewed.

Open Access This is an Open Access article distributed in accordance with the Creative Commons Attribution Non Commercial (CC BY-NC 3.0) license, which permits others to distribute, remix, adapt, build upon this work noncommercially, and license their derivative works on different terms, provided the original work is properly cited and the use is non-commercial. See: http://creativecommons.org/licenses/by$\mathrm{nc} / 3.0 /$

\section{REFERENCES}

1 Kohn LT, Corrigan JM, Donaldson MS. To Err is Human: Building a Safer Healthcare System. Medicine Io, ed. https:// download.nap.edu/catalog.php?record_id $=97282000$

2 Vincent $\mathrm{C}$, Neale G, Woloshynowych $\mathrm{M}$. Adverse events in British hospitals: preliminary retrospective record review. BMJ 2001;322:517-19.

3 de Vries EN, Ramrattan MA, Smorenburg SM, et al. The incidence and nature of in-hospital adverse events: a systematic review. Qual Saf Health Care 2008;17: 216-23.

4 Cullinane M. Scoping our practice: The 2004 Report of the National Confidential Enquiry into Patient Outcome and Death. National Confidential Enquiry into Perioperative Deaths, London, 2004.

5 Gavin DR, Valori RM, Anderson JT, et al. The national colonoscopy audit: a nationwide assessment of the quality and safety of colonoscopy in the UK. Gut 2013;62:242-9.

6 UK BC. Bowel Cancer UK: Improving capacity saving ilves: Endoscopy in the UK.

7 Kwan V. Advances in gastrointestinal endoscopy. Intern Med J 2012;42:116-26.

8 The Patient Safety Policy Team. The "never events" list 2012/ 13. In: Health Do, ed. http://www.dh.gov.uk/health/2012/01/ never-events-update/2012

9 Reason J. Human error: models and management. BMJ 2000;320:768-70.

10 Sevdalis N, Hull L, Birnbach DJ. Improving patient safety in the operating theatre and perioperative care: obstacles, interventions, and priorities for accelerating progress. $\mathrm{Br} J$ Anaesth 2012;109(Suppl 1):i3-i16.

11 Flin R. Leadership for safety: industrial experience. Qual Saf Health Care 2004;13(Suppl_2):ii45-51.

12 Haynes AB, Weiser TG, Berry WR, et al. A surgical safety checklist to reduce morbidity and mortality in a global population. N Engl J Med 2009;360:491-9.

13 Walker IA, Reshamwalla S, Wilson IH. Surgical safety checklists: do they improve outcomes? Br J Anaesth 2012;109:47-54.

14 Verdaasdonk EG, Stassen LP, Widhiasmara PP, et al. Requirements for the design and implementation of checklists for surgical processes. Surg Endosc 2009;23:715-26.

15 Ranji SR, Shojania KG. Implementing patient safety interventions in your hospital: what to try and what to avoid. Med Clin North Am 2008;92:275-93, vii-viii.

16 Parand A, Benn J, Burnett S, et al. Strategies for sustaining a quality improvement collaborative and its patient safety gains. Int J Qual Healthcare 2012;24:380-90. 
17 Vats A, Vincent CA, Nagpal K, et al. Practical challenges of introducing WHO surgical checklist: UK pilot experience. BMJ 2010;340:b5433.

18 Fourcade A, Blache JL, Grenier C, et al. Barriers to staff adoption of a surgical safety checklist. BMJ Qual Saf $2012 ; 21: 191-7$.
19 Valori RBR. BSG Quality and Safety Indicators for Endoscopy. GI Endoscopy Publication for the Joint Advisory Group, 2007.

20 Sint Nicolaas J, de Jonge V, de Man RA, et al. The Global Rating Scale in clinical practice: a comprehensive quality assurance programme for endoscopy departments. Dig Liver Dis 2012;44:919-24. 